\title{
Detection of pneumothorax by accident and emergency officers and radiologists on single chest films
}

\author{
F. AITCHISON*, A. BLEETMAN ${ }^{\dagger}$, P. MUNRO ${ }^{+}$, D. \\ MCCARTER* \& A. W. REID* \\ ${ }^{*}$ Department of Radiology and ${ }^{+}$Accident and Emergency Department, Glasgow Royal \\ Infirmary, Alexandra Parade, Glasgow
}

\section{SUMMARY}

To assess whether an accurate diagnosis of pneumothorax can be made on a single chest film, 233 pairs of inspiratory (I) and expiratory (E) chest films taken in an accident and emergency (A\&E) department for suspected pneumothorax were reviewed by two $A \& E$ officers and three radiologists. The films were assessed for the presence of pneumothorax by viewing the I film in isolation and, after an interval, by viewing the paired I and E films together. Fifty-four of the patients had a pneumothorax. The five observers missed 23 pneumothoraces $(8.5 \%$ of total) on the I film alone which were correctly diagnosed on the paired I and E films, the three radiologists missed 10/162 pneumothoraces on the I film alone which were correctly identified on the I and E films (6\%) and the two A\&E officers 13/108 $(12.5 \%)$. The use of a single inspiratory chest film for suspected pneumothorax could result in pneumothoraces being missed, particularly by less experienced observers and therefore we believe that paired I and E films should continue to be used routinely for suspected pneumothorax.

\section{INTRODUCTION}

Paired I and E chest films are taken in many hospitals as the standard investigation for suspected pneumothorax. An I film allows the best assessment of the lungs and the bony thorax. The E film is believed to make a pneumothorax more obvious. In an $\mathrm{E}$ film the volume of air in the pleural cavity is relatively greater in relation to

Correspondence: $\operatorname{Dr}$ F. Aitchison, Departnent of Radiology, Glasgow Royal Infirmary, Alexandra Parade Glasgow. 
the volume of the lung (Green et al., 1977) and the volume of air in the lung is reduced which makes the lung more dense and the lung edge more obvious, (Blank, 1989).

Bradley et al. (1991), after a study by two radiologists, suggested that E films should not be performed routinely in the diagnosis of pneumothorax. By routinely taking only I films the cost of the investigation and radiation dose to the patient would be reduced.

Clinical decisions in the $A \& E$ department are often based on X-ray film interpretation by junior A\&E officers. To our knowledge there has been no previous study to compare the detection rate of pneumothorax on I films alone against the detection rate on paired I and E films by observers with different levels of experience.

\section{METHODS}

Two hundred and thirty-three pairs of $\mathrm{I}$ and $\mathrm{E}$ chest radiographs taken at our hospital between January 1988 and September 1991 were reviewed. The original examinations had all been performed in the $A \& E$ department for suspected pneumothorax. Patients were aged 17-81 years (mean age 39 years) and 161 (69\%) were male. Fifty-four patients had a pneumothorax.

Each inspiratory films was assessed in isolation by five independent observers for the presence of a pneumothorax. After an interval of at least 8 weeks the inspiratory and expiratory films were assessed together. Films were viewed in batches of 25 single films or 25 pairs of I and E films. If a pneumothorax was considered present the side and size were recorded. The size was assessed subjectively as small, medium or large (Blank, 1989).

The observers were two A\&E senior house officers, two radiology registrars and one radiology consultant.

The 54 patients with pneumothorax were aged between 17 and 63 years (mean age 34 years), 41 were male $(76 \%)$. There were 30 left-sided pneumothoraces and 24 right-sided pneumothoraces (no statistically significant difference).

Differences of opinion were resolved by consensus.

\section{RESULTS}

Of the total 270 instances where a pneumothorax should have been identified by the five observers (54 pneumothoraces $\times 5), 23(8.5 \%)$ were missed on the I film but correctly identified on the paired I and E films. The three radiologists missed $10 / 162$ pneumothoraces $(6 \%)$ on I film alone but not on I and E film and the two A\&E officers missed $13 / 108$ pneuothoraces (12.5\%) on I alone but not on I and E film.

A total of $16 / 108$ pneumothoraces were missed by the two A\&E officers on both I and I and E films (14.8\%) and 4/162 pneumothoraces were missed on both I and $\mathrm{I}$ and $\mathrm{E}$ films by the three radiologists $(2.4 \%)$. 
Each of the five observers made fewer errors using paired I and E films than using I films alone. In total there were 34 comparisons between I film alone and I and E film assessments where an individuals assessment based on I film alone differed from their assessment using I and E films. In 29 of these 34 instances $(85 \%)$ it was the I and $\mathrm{E}$ assessment which was correct.

Clearly, and not unexpectedly the performance of the radiologists was superior to that of the A\&E officers. There were 139 instances where an A\&E officer's assessment differed from a radiologists assessment, and in 131 of these 139 instances (94\%) it was the radiologist who was correct.

There were two patients with pneumothorax which were both missed on the I film alone by all five observers but detected by two radiologists on the paired I and E films.

All of the pneumothoraces which were missed were graded small by the other observers.

On review of the films considered false positive, four showed a prominent apical pleural cap (soft tissue band at lung apex). Rather surprisingly no-one mistook several large bullae for pneumothoraces.

\section{DISCUSSION}

The detection of a pneumothorax will usually alter patient management and it is important the diagnosis is made. In this study the pneumothorax detection rate increased by $8.5 \%$ overall by using paired I and E films compared with I films alone and for less experienced observers (A\&E officers) the pneumothorax detection rate increased by $12.5 \%$. We believe that it would be unwise to perform I films routinely for suspected pneumothorax only, particularly if the assessment is made by relatively inexperienced staff because of the number of pneumothoraces which could be missed.

\section{CONCLUSION}

Routine I and E chest films should continue to be performed in the A\&E department for suspected pneumothorax.

\section{ACKNOWLEDGEMENTS}

We would like to thank Miss Angela Gruner for typing the manuscript and to acknowledge the help of Dr Christopher Moulten and Dr Gordon Murray, Reader in Medical Statistics, Glasgow Royal Infirmary. 


\section{F. Aitchison et al.}

\section{REFERENCES}

Blank N. (1989) Che'st Radiographic Analysis, pp. 77-79. Churchill Livingston Inc., New York.

Bradley M., Williams C. \& Walshaw M. J. (1991) The value of routine expiratory chest films in the diagnosis of pneumothorax. Archioes of Emergency Medicine 8, 115-116.

Greene R., McLoud T. C. \& Stark P. (1977) Pneumothorax. Seminars in Roentgenology XII, $313-324$.

O'Carroll T. (1985) Complications during anaesthesia In: Textbook of Anaesthesia (eds Smith G. \& Aitkenhead A. R.). pp. 278. Churchill Livingston, Edinburgh. 